\title{
The selection and prevalence of natural and fortified calcium food sources in the diets of adolescent girls
}

\author{
Karen Rafferty, RD, LMNT, \\ Senior Research Dietitian, Osteoporosis Research Center, Creighton University \\ Patrice Watson, PhD, and \\ Statistician and Data Manager, Osteoporosis Research Center, Creighton University, Omaha, NE \\ 68131
}

Joan M. Lappe, PhD, RN

Professor of Medicine and Nursing, Creighton University, Omaha, NE 68131

\begin{abstract}
Objective-To assess the impact of calcium-fortified foods and dairy foods on selected nutrient intakes in the diets of adolescent girls.

Design-Randomized controlled trial, secondary analysis.

Setting and Participants-149 adolescent girls from a Midwestern metropolitan area participated in randomized controlled trials of bone physiology from 1997 to 2008.

Intervention-Subjects randomly assigned to a high calcium (HC) diet supplying $1500 \mathrm{mg}$ calcium/day, or their usual diet (UC).

Main Outcome Measures-Dietary intake was assessed from 3-day food records and calcium intakes categorized by food source. Food group composites, representing calcium-fortified and dairy food categories, were examined for their relative nutrient contributions. Students $t$ tests were used to evaluate differences in selected nutrient intakes between the two study groups.
\end{abstract}

Results-Dairy foods contributed 68\% of the total mean $1494 \mathrm{mg}$ calcium/d in the HC group, and calcium-fortified foods contributed $304 \mathrm{mg}$ calcium. In the UC group dairy foods contributed $69 \%$ of the total mean $765 \mathrm{mg}$ calcium/d and calcium-fortified foods contributed $50 \mathrm{mg} \mathrm{Ca}$. Nutrient profiles of the dairy composites differed significantly from the calcium-fortified composites $(\mathrm{p}<0.05)$.

Conclusions and Implications-Dairy foods were the primary source of calcium selected by these adolescent girls; calcium-fortified foods augmented calcium intakes.

\section{Keywords}

calcium; dairy foods; calcium-fortified foods; nutrient profiles; nutrition surveillance

\footnotetext{
Corresponding Author: 601 N. $30^{\text {th }}$ Street, Suite 4820, Omaha, NE 68131, Phone: 402.280.4680; Fax: 402.280.5034, karenrafferty@creighton.edu.

The authors have no conflict of interest to declare.

Publisher's Disclaimer: This is a PDF file of an unedited manuscript that has been accepted for publication. As a service to our customers we are providing this early version of the manuscript. The manuscript will undergo copyediting, typesetting, and review of the resulting proof before it is published in its final citable form. Please note that during the production process errors may be discovered which could affect the content, and all legal disclaimers that apply to the journal pertain.
} 


\section{INTRODUCTION}

Over the last several decades the rise in osteoporotic bone disease has reached epidemic proportions (1). Consequently, there has been great emphasis on promoting bone health, particularly in growing children who are developing the skeletons that need to last for their lifetime. Optimal calcium intake is recognized to be a critical factor in the development and maintenance of a healthy skeleton. Other nutrients identified as bone-related nutrients by the Institute of Medicine (IOM), namely phosphorus, magnesium, and vitamin D, also play a key role in the development and maintenance of bone tissue (2). Adequate protein and potassium have been shown to favor bone health as well $(3,4)$. Milk and milk products contribute significant levels of calcium and other essential nutrients to the nation's food supply (5). Meeting dietary calcium recommendations without consuming dairy foods is difficult $(2,6,7,8)$ and studies show that diets low in calcium and dairy foods tend to be low in multiple nutrients $(9,10)$. However, $77 \%$ of U.S. children age 9-19 do not consume the recommended 3 servings/day from the milk and milk products food group (11) and nearly $90 \%$ of U.S. teenage girls do not meet the calcium adequate intake (AI) of $1300 \mathrm{mg} / \mathrm{day}$ (12). In recent years calcium-fortified foods have been suggested as an alternative calcium source for achieving adequate dietary calcium intakes $(13,14)$.

Few foods were fortified with calcium in the early 1990's (15), but in the ensuing years there has been a steady increase in the availability of calcium-fortified foods (16). From 1999 to 2006 over 1100 new or re-formulated calcium-fortified foods entered the market $(17,18)$. Calcium-fortified food categories include fruit and juice beverages, breakfast cereals, cereal and snack bars, soy beverages, pasta products, breads, margarines, chocolate syrup, and even soft drinks and bottled "fitness" waters $(18,19)$. Commenting on the growing number of calcium-fortified foods available to consumers as early as 1997, the IOM cautioned that it is important to "maintain surveillance of the calcium-fortified products in the marketplace and to monitor their impact on calcium intake" (2). There is, however, little published research on the impact of discretionary calcium fortification practices on total dietary calcium intakes of the U.S. population (15). The purpose of this analysis was to examine and quantify the food sources of calcium in the self-selected diets of teenage girls who participated in two randomized controlled trials of bone physiology from 1997-2008. An additional objective was to develop composites for the calciumfortified food category and the dairy food category, along with their associated nutrient profiles, in order to identify intakes of the bone-related nutrients that can be expected from consuming a specific calcium load from dairy foods and from calcium-fortified foods. We postulated that the nutrient profile of the calcium-fortified foods as consumed by our study population would differ significantly from the nutrient profile of dairy foods. To our knowledge, this is the first report that attempts to quantify the penetration and nutritional impact of calcium-fortified foods in this segment of the population.

\section{METHODS}

\section{Study Design}

This was a secondary analysis of data obtained from two randomized controlled trials testing the bone mass effect of increasing dietary calcium intake to $1,500 \mathrm{mg} /$ day in pubertal girls. In the first study, beginning in 1997, a convenience sample of 64 healthy 9-year-old girls was recruited from a metropolitan area and randomly assigned to one of two groups: (a) a high-calcium treatment group instructed to consume calcium-rich foods to supply at least $1,500 \mathrm{mg} / \mathrm{day}$; and (b) a control group instructed to continue their usual diet. The following year, a similar cohort of 134 healthy 9-year old girls was recruited for a second experimental study and randomized to one of three groups: (a) a high-calcium treatment group assigned to an weight-bearing exercise regimen in addition to a calcium-rich diet supplying at least 
$1,500 \mathrm{mg}$ calcium/day; (b) a treatment group assigned to a weight-bearing exercise regimen asked to continue their usual diet; and (c) a control group asked to continue their usual diet and exercise patterns. All subjects were asked to refrain from using calcium supplements. Dietary intake was assessed on all subjects at baseline and every 3 months for 10 years (Study 1) and 8 years (Study 2). The dietary data presented in this secondary analysis were collected and averaged over a 36 month period, commencing in 2002 and concluding in 2005 , from that subset of subjects who remained on study through $2005(n=149)$.

\section{Subjects and setting}

Subjects were recruited through the Great Plains Girls Scout Council and metropolitan area schools. Children with a history of lactose intolerance, milk allergy, corticosteroid or anticonvulsant therapy, or any other significant health problem identified by the parent(s) were excluded. We also excluded girls who had usual dietary intake of more than $1,100 \mathrm{mg}$ calcium/day and a body mass index (BMI) in the $85^{\text {th }}$ percentile or more for age and sex $(B M I \geq 20)$. All of the girls were premenarcheal and in Tanner Stage 1 upon entry into the studies. The studies were approved following full review by the Creighton University Institutional Review Board. Signed informed assent was obtained from all subjects and signed informed consent from at least one parent of each girl.

Subjects came to the Osteoporosis Research Center for all study visits. The present analysis was applied to data collected from 149 girls who completed 7 or more years on study: 55 on high calcium diets (one non-Hispanic black, two Hispanic white and 52 non-Hispanic white) and 94 on their usual diets (two non-Hispanic black and 92 non-Hispanic white.) 49 subjects enrolled in the original studies either moved from the area or dropped from the studies before completing the $7^{\text {th }}$ year and are not included in this secondary analysis. At the end of year 7 the girls were $15-16$ years of age.

\section{Dietary Intervention}

Subjects assigned to the high-calcium treatment groups (HC) were instructed to consume $1,500 \mathrm{mg}$ calcium per day from food sources. Both dairy foods and calcium-fortified nondairy foods were promoted as acceptable sources of dietary calcium, and the girls were free to select foods from both sources according to their personal preferences. The girls and their parent(s) were educated about dairy and calcium-fortified food sources and taught to interpret the calcium content of foods from the Nutrition Facts panel. For the purposes of these trials, a calcium-fortified food was considered an acceptable calcium source if it provided a minimum of $100 \mathrm{mg}$ calcium per serving (10\% Daily Value on the Nutrition Facts panel.) To cover the costs of their calcium foods, the families were provided with a customized credit card for a local supermarket chain and did not incur any direct out-ofpocket expenses. The supermarket then billed our research center for the charges. The average expenditure for the purchase of the dairy and/or calcium-fortified foods was maintained at around $\$ 13$ per family per week.

Subjects assigned to the usual diet groups (UC) were instructed to continue with their usual diets. The importance of overall good nutrition was discussed and each girl received a copy of the Food Guide Pyramid Guide to Good Eating (20).

\section{Dietary Assessments}

Nutrient intake was assessed from 3-day diet records completed for two weekdays and one weekend day. The subjects and their parent(s) were instructed by the registered dietitian to record weighed and/or measured amounts of all foods and beverages consumed, to use product brand names when recording dietary intake, and to specify if any items consumed were calcium-fortified. Food models were used to help the subjects determine portion sizes. 
Both the $\mathrm{HC}$ and the UC subjects completed the 3-day diet diaries every 3 months for a maximum of 36 days of dietary intake records (mean 30; range 24-36) completed during the three-year assessment period of this secondary analysis, a compliance rate of $83 \%$. Nelson et al (21) reported that 19 days of dietary records are sufficient to ensure $r \geq 0.9$ for energy, protein, carbohydrate, sugars, fat, calcium, magnesium, phosphorus, and potassium in girls age 5-17. Diet records were reviewed as they were turned in to confirm that all written food items were legible and to clarify the amounts of foods consumed. The registered dietitian analyzed all diet records for nutrient composition and dietary sources of calcium using The Food Processor Nutrition and Fitness Software (Version 7.8, 2001, ESHA Research, Salem, OR). Maintaining an up-to-date database of calcium-fortified foods was an on-going process during the decade-long course of the original studies. In general, the ESHA database values for fortified nutrients such as calcium are the values calculated by the food manufacturer, or by the USDA Nutrient Data Laboratory based on the Nutrition Facts panel statement of \% Daily Value (DV) (22). Database calcium values were also updated periodically by the dietitian using information from food product Nutrition Facts panels, to ensure that up-todate nutrient values of calcium-fortified foods were maintained. Table 1 presents an example of changes in the USDA Nutrient Database for Standard Reference, reflecting changes in calcium fortification levels in selected cereal grain products concurrent with the time period of these trials.

Calcium food sources were categorized by the dietitian as dairy, calcium-fortified non-dairy, or non-dairy/non-fortified other. Dairy sources of calcium included milk ( $2 \%$ fat, $1 \%$ fat, and non-fat fluid plain milk; flavored milk; milk used in soup, pudding, and latte/coffee beverages); cheese (natural and processed cheese; as ingredient in sandwiches, pizza, and menu items such as cheeseburgers, tacos; and cheese in cooked dishes such as macaroniand-cheese, lasagna); yogurt; and ice cream. Calcium-fortified foods were tabulated as calcium-fortified fruit juice (100\% fruit juices); calcium-fortified ready-to-eat (RTE) breakfast cereals; calcium-fortified cereal/granola bars; and calcium-fortified breads. A pooled category named "calcium-fortified miscellaneous" consisted of various calciumfortified foods consumed in low volume/low frequency during the assessment period (e.g. calcium-fortified varieties of: waffles, pasta products, margarines, fruit snacks, milk flavorings, soy beverages, meal-replacements, and fruit-flavored beverages.) Finally, the non-dairy/non-fortified other category included natural calcium from vegetables, fruits, meat, poultry, fish, legumes, grain products, snacks, and candies. A composite approach was used to determine the nutrient composition of the calcium-fortified foods and the dairy foods as consumed by the $\mathrm{HC}$ and the UC groups. The calcium-fortified and dairy composites were based on actual food and beverage intakes as reported by the subjects, and reflected the proportional calcium contribution of individual food items within the two categories. Profiles were then developed for each of the composites for energy, protein, carbohydrate, fat, added sugar, sodium, calcium, phosphorus, potassium, vitamin A, vitamin $\mathrm{D}$, and magnesium. Nutrient profiles were adjusted to an equivalent calcium load of $300 \mathrm{mg}$ calcium. This adjusted value allowed for comparison of the calcium-fortified composite and the dairy composite nutrient profiles. The Food Processor Nutrition and Fitness Software, previously described, was used to calculate the adjusted nutrient profiles based on the proportional contribution of each food component represented in the composites.

\section{Data analysis}

Data were analyzed with MS Excel spreadsheet and SPSS for Windows (Version 11, 2003, SPSS Inc, Chicago, IL). Descriptive statistics and Students $t$ tests were used to evaluate differences in selected nutrient intakes between the two study groups (SAS Version 9, SAS Institute, Cary, NC.) A significance level of $p<0.05$ was used for all analyses. 


\section{RESULTS}

In the HC group and the UC group, dairy foods contributed $68 \%$ and $69 \%$ of the total calcium respectively. The mean calcium intake in the $\mathrm{HC}$ group was $1494 \pm 551 \mathrm{mg} / \mathrm{d}$, and the top three contributors of calcium were milk (45\%), cheese (17\%), and calcium-fortified foods $(20 \%)$. $95 \%$ of the girls in the HC group selected a calcium-fortified food at least once during the 36 month assessment period. Calcium-fortified fruit juice contributed $6.7 \%$ of the total calcium, followed by calcium-fortified RTE breakfast cereals (6.6\%), and calciumfortified cereal bars $(3.2 \%)$.

In the UC group (mean $765 \pm 197 \mathrm{mg}$ calcium/d), the top three contributors of calcium were milk (39\%), cheese (25\%), and non-dairy/non-fortified other foods (23\%). Although $97 \%$ of the girls in this group reported selecting a calcium-fortified food at least once during the assessment period, calcium-fortified foods contributed only $6.6 \%$ of the total calcium. Calcium-fortified RTE breakfast cereals, the most prevalent calcium-fortified food reported in the UC group, contributed $2.5 \%$ of the total dietary calcium, followed by calciumfortified fruit juice (1.5\%) and calcium-fortified cereal bars (1.0\%). In the HC group, dairy foods contributed a mean $1014 \pm 439 \mathrm{mg}$ calcium/d, the calcium equivalent of 3.4 cups of milk and milk products. Calcium-fortified foods contributed $304 \pm 258 \mathrm{mg}$ calcium $/ \mathrm{d}$. The UC group reported a mean $539 \pm 175 \mathrm{mg}$ calcium/d from dairy foods (the equivalent of 1.8 cups of milk and milk products), and a mean $50 \pm 47 \mathrm{mg}$ calcium/d from calcium-fortified foods. The differences in absolute total calcium, calcium contributed by dairy foods, and calcium contributed by calcium-fortified foods were significant between the HC and UC groups $(\mathrm{p}<.001)$. Calcium contributed by the non-dairy/non-fortified other category was $176 \pm 50 \mathrm{mg} / \mathrm{d}$ for the HC group and $176 \pm 48 \mathrm{mg} / \mathrm{d}$ for the UC group (NS).

Based on the mean proportionate distribution of calcium from dairy foods reported by the HC and UC groups, respective composites for the dairy category were developed. In similar fashion, composites were developed for the calcium-fortified foods. Table 2 identifies the proportionate contribution of the various food items to each composite's total calcium. Nutrient profiles for the dairy and calcium-fortified categories, based on these composites and adjusted to an equivalent $300 \mathrm{mg}$ calcium load, are set forth in Figure 1 for the HC group. (The nutrient profiles developed from the dairy and calcium-fortified composites of the UC group were not significantly different from the nutrient profiles of the $\mathrm{HC}$ group; therefore the UC data is not shown.)

\section{DISCUSSION}

The aim of this analysis was to examine the selection, prevalence, and relative nutritional contribution of dairy and calcium-fortified foods in the diets of a cohort of adolescent girls. Although this was a randomized controlled intervention trial with one group (HC) receiving regular and explicit information to increase dietary calcium, the food sources of calcium were nevertheless independently selected by each subject based on personal preference. Therefore this analysis is useful in quantifying the impact of calcium-fortified foods in selfselected diets within the context of regular dietary counseling to maintain a high calcium intake, and monetary reimbursement to cover the cost of the high calcium foods. In contrast, the lesser impact of calcium-fortified foods as selected by the UC group can be considered to be more representative of the general population of adolescent girls consuming their usual diets.

The mean calcium intake of the UC group was approximately $75 \mathrm{mg} / \mathrm{d}$ lower than the 20052006 NHANES national average of for this age group (12). However, NHANES data include calcium consumed as dietary supplements (23), not included in the present analysis 
because none of our subjects reported consumption of supplemental calcium. Irrespective of the absolute calcium content of the HC and UC self-selected diets, both groups were consistent with data reported by others showing $65-70 \%$ of total dietary calcium supplied by dairy foods (24). $17 \%$ to $25 \%$ of total dietary calcium was contributed by cheese in the HC and UC groups, a finding that is also consistent with national survey data for adolescent girls $(25,26)$. Thus, calcium-fortified foods did not appear to displace dairy foods in the diets of our study population.

The top contributor of calcium from a calcium-fortified food source for the total cohort was calcium-fortified RTE breakfast cereal. RTE breakfast cereal has been a vehicle for nutrient enrichment and fortification for decades (15). Calcium-fortified RTE breakfast cereal contributed approximately $2-3 \%$ of the mean daily calcium in the UC group, consistent with NHANES 1999-2002 national survey data (27).

At equivalent $300 \mathrm{mg}$ calcium loads, the calcium-fortified nutrient profile differed from the nutrient profile of the dairy foods. A greater contribution of energy, carbohydrate, added sugar, and sodium and a lesser contribution of protein, phosphorus, potassium and vitamin A from calcium-fortified composite was found. We also found that the calcium-fortified composite reflected a higher contribution of magnesium and vitamin $\mathrm{D}$, and a lesser contribution of total fat, relative to an equivalent $300 \mathrm{mg}$ calcium load from the dairy composite. There are two potential explanations for this occurrence. Both magnesium and vitamin D were supplied in the calcium-fortified composite by RTE breakfast cereals and fruit juices, many of which were fortified with vitamin D, naturally occurring sources of magnesium, and low in fat. Secondly, the dairy food composite reflected a proportional contribution from cheese representing $25-35 \%$ of the total dairy calcium. Relative to fluid milk, cheese has a lower magnesium content and a higher fat content and, unlike milk, is not fortified with vitamin D. Consequently, the dairy composite contributed lower values for magnesium and vitamin $\mathrm{D}$ and higher values for fat than did the calcium-fortified composite at an equivalent calcium load. A higher fat value, however, did not result in a higher energy value. An increased value for carbohydrate and added sugar in the calcium-fortified composite contributed to an energy value of 211 calories; 36\% higher than the 155 calorie energy value of the dairy food composite.

Data from NHANES 2005-2006 indicate that calcium, phosphorus, magnesium, potassium, and vitamin D remain nutrients of concern for teenage girls (12). The Dietary Guidelines for Americans (DGA) recommendation of three cups of milk and milk products/d was designed to provide $69 \%$ of the calcium, $56 \%$ of the phosphorus, $26 \%$ of the potassium, and $23 \%$ of the magnesium recommended for this age group, in addition to $54 \%$ of the protein and $61 \%$ of the vitamin A (6). The HC group achieved each of these target nutrient adequacy goals exclusively from dairy foods. Thus, while it is useful to compare nutrient profiles of the calcium-fortified and dairy composites at equivalent calcium levels, such a comparison does not reflect total nutrient intakes or overall dietary adequacy. Due to a preference for dairy foods as a dietary calcium source, the HC group met or exceeded the nutrient adequacy values intended to be achieved by the recommended 3 cups of milk and milk products/d for calcium and each of the nutrients measured.

\section{Implications for Nutrition Education}

Calcium-fortified foods have been identified as an alternative calcium source for individuals who cannot or choose not to consume milk and milk products $(13,14)$, or as a means of augmenting low calcium intakes $(16,19)$. However, studies have shown that individuals who obtain their calcium from non-dairy sources fail to meet the nutrient profile of those who consume dairy foods $(6,7,9,10,17)$. This study confirms those findings. 
It should be emphasized that calcium-fortified foods do not constitute a sub-group within the milk and milk products food group, and it is therefore inappropriate to position calciumfortified foods as a nutritionally equivalent alternative to milk and milk products in public health policy or in nutrition education settings. At the present time there is not enough evidence available on the population-wide impact of calcium-fortified foods on overall nutrient adequacy, diet quality, or health outcomes to support recommending these foods as dairy alternatives. Furthermore, foods supplying calcium in amounts equivalent to dairy foods cannot be assumed to deliver equivalent calcium bioavailability. A comprehensive analysis of studies measuring the bioavailability of calcium carbonate as a fortificant reveals a span of mean fractional calcium absorption values ranging from 0.21 to 0.42 (28). Such disparity compounds the challenge faced by nutrition professionals of accurately assessing the impact of calcium-fortified foods on calcium nutrition.

Nutritionists and health educators should be aware of the total nutrient contributions of calcium-fortified foods relative to dairy foods and tailor nutrition messages to consumers accordingly, addressing total energy intakes and nutrient adequacies beyond calcium. Our data confirm reports from others concluding that incorporating milk and milk products into the diets of adolescent girls is the best way to achieve adequate intakes of calcium and other bone-related nutrients $(8,9,10,17)$. For those who avoid milk and milk products, dietary guidance must consider strategies for replacing not only calcium but other significant nutrients supplied by milk as well, within appropriate energy levels.

\section{Implications for Nutrition Surveillance}

In nutrition surveillance data collected 1989-1991 only $2.5 \%$ of the population of teenage girls reported using a calcium-fortified food at least once (15). Our data, gathered 10-15 years later, identified $96 \%$ of the total cohort as having selected a calcium-fortified food at least once. Thus, over the span of one decade, the penetration of calcium-fortified foods in the consumer market has become nearly ubiquitous.

The increased availability of calcium-fortified foods underscores the importance of accurately assessing calcium intakes and the impact of calcium-fortified foods on nutrient intakes and diet quality in the population. A comprehensive national database accurately reflecting available calcium-fortified foods must be continually updated, consistent with changes in the food supply (29). Dwyer et al cite a critical need for enhanced capability to identify calcium-fortified foods in national surveys and to assess nutrient intakes from fortified food sources (23). Thus, nationwide calcium intake data may currently underestimate intakes due to erratic market trends in calcium fortification of foods and delays in updating databases (30).

\section{Limitations}

Several limitations must be acknowledged. The sample size of our study was small and limited to a regional population of primarily Caucasian adolescent girls. Larger studies are needed to elucidate the impact of calcium-fortified foods on total calcium intakes, diet quality, and nutrient adequacies across the full range of ages and ethnicities, in men and women. Secondly, it was beyond the scope of this analysis to examine the contribution of dairy and calcium-fortified foods on total diet quality and nutrient intake from all dietary sources. Therefore, while our data suggest that calcium-fortified foods do not constitute a nutritionally equivalent substitute for milk and milk products, nutrient shortfalls cannot be predicted unless total dietary intake is taken into account. Nevertheless, the information gleaned from following this group of urban females gives us a better understanding of the food sources supplying calcium in the diets of contemporary adolescent girls and 
underscores the need to monitor the impact of calcium-fortified foods on total calcium intakes of the U.S. population.

\section{Acknowledgments}

Funding sources and acknowledgements: This research was supported in part by funds from the National Institute of Child Health and Human Development (grant \# HD36601), the National Institute of Nursing Research (grant \# NR004336) and the National Dairy Council. The authors thank Julie Stubby, R.N., B.S.N, and Gina Lypaczewski, R.N., $\mathrm{MSc}(\mathrm{A})$, project managers for the experimental studies from which the data presented in this analysis were generated.

This research was conducted while the authors were affiliated with the Osteoporosis Research Center of Creighton University in Omaha, Nebraska.

The original experimental studies from which these data were generated were approved following full review by the Creighton University Institutional Review Board.

\section{References}

1. U.S. Department of Health and Human Services. Bone Health and Osteoporosis: A Report of the Surgeon General. Rockville, MD: U.S. Department of Health and Human Services, Office of the Surgeon General; 2004.

2. Institute of Medicine. Dietary Reference Intakes for Calcium, Phosphorus, Magnesium, Vitamin D, and Fluoride. Washington, D.C: National Academies Press; 1997.

3. Institute of Medicine. Dietary Reference Intakes for Energy, Carbohydrate, Fiber, Fat, Fatty Acids, Cholesterol, Protein, and Amino Acids. Washington, D.C: National Academies Press; 2002.

4. Institute of Medicine. Dietary Reference Intakes for Water, Potassium, Sodium, Chloride, and Sulfate. Washington, D.C: National Academies Press; 2004.

5. Rafferty KA, Heaney RP. Nutrient effects on the calcium economy: emphasizing the potassium controversy. J Nutr 2008;138:166S-171S. [PubMed: 18156419]

6. Dietary Guidelines Advisory Committee. Report of the Dietary Guidelines Advisory Committee on the Dietary Guidelines for Americans, 2005. Washington DC: U.S. Department of Agriculture, Agricultural Research Service; 2004.

7. Gao X, Wilde PE, Lichtenstein AH, Tucker KL. Meeting adequate intake for dietary calcium without dairy foods in adolescents aged 9 to 18 years (National Health and Nutrition Examination Survey 2001-2002). J Am Diet Assoc 2006;106:1759-1765. [PubMed: 17081826]

8. Weaver CM. Back to basics: have milk with meals. J Am Diet Assoc 2006;106:1756-1758. [PubMed: 17081825]

9. Nicklas TA, O'Neil CE, Fulgoni VL. The role of dairy in meeting the recommendations for shortfall nutrients in the American diet. J Am Coll Nutr 2009;28:73S-81S. [PubMed: 19571165]

10. Heaney RP. Dairy and bone health. J Am Coll Nutr 2009;28:82S-90S. [PubMed: 19571166]

11. National Dairy Council. Taking action to improve children's nutrition and fitness: the role of schools. Dairy Council Digest 2003;7:1-6.

12. Moshfegh, A.; Goldman, J.; Ahuja, J.; Rhodes, D.; LaComb, R. What We Eat in America, NHANES 2005-2006: Usual Nutrient Intakes from Food and Water Compared to 1997 Dietary Reference Intakes for Vitamin D, Calcium, Phosphorus, and Magnesium. U.S. Department of Agriculture, Agricultural Research Service; 2009 [Accessed July 24, 2009.]. http://www.ars.usda.gov/ba/bhnrc/fsrg

13. Greer FR, Krebs NF. the Committee on Nutrition. Optimizing bone health and calcium intakes of infants, children, and adolescents. Pediatrics 2006;117:578-585. [PubMed: 16452385]

14. American Dietetic Association. Position of the American Dietetic Association: Nutrient Supplementation. J Am Diet Assoc 2009;109:2073-2085. [PubMed: 19957415]

15. Berner LA, Clydesdale FM, Douglass JS. Fortification contributed greatly to vitamin and mineral intakes in the United States, 1989-1991. J Nutr 2001;131:2177-2183. [PubMed: 11481414] 
16. Forshee RA, Anderson PA, Storey ML. Changes in calcium intake and association with beverage consumption and demographics: comparing data from CSFII 1994-1996, 1998 and NHANES 1999-2002. J Am Coll Nutr 2006;25:108-116. [PubMed: 16582026]

17. Miller GD, Jarvis JK, McBean LD. The importance of meeting calcium needs with foods. J Am Coll Nutr 2001;20:168S-185S. [PubMed: 11349940]

18. Clark, P. Mintel Global New Products Database Search. Mintel International Group Ltd; Chicago, Ill., USA: 2006. Unpublished data

19. Olson BH, Chung KR, Reckase M, Schoemer S. Parental influences on dairy intake in children, and their role in child calcium-fortified food use. J Nutr Educ Behav 2009;41:53-58. [PubMed: 19161921]

20. National Dairy Council. Guide to Good Eating ( ). National Dairy Council; Rosemont IL: 1992.

21. Nelson M, Black AE, Morris JA, Cole TJ. Between- and within-subject variation in nutrient intake from infancy to old age: estimating the number of days required to rank dietary intakes with desired precision. Am J Clin Nutr 1989;50:155-167. [PubMed: 2750688]

22. U.S. Department of Agriculture, Agricultural Research Service. USDA National Nutrient Database for Standard Reference, Release 22. Nutrient Data Laboratory Home Page. 2009 [Accessed July 2, 2009.]. http://www.ars.usda.gov/ba/bhnrc/ndl

23. Dwyer J, Picciano MF, Raiten DJ. Members of the Steering Committee. Collection of food and dietary supplement intake data: What We Eat in America-NHANES. J Nutr 2003;133:590S-600S. [PubMed: 12566509]

24. Fiorito LM, Mitchell DC, Smiciklas-Wright H, Birch LL. Dairy and dairy-related nutrient intake during middle childhood. J Am Diet Assoc 2006;106:534-542. [PubMed: 16567149]

25. Albertson AM, Tobelmann RC, Marquart L. Estimated dietary calcium intake and food sources for adolescent females: 1980-92. J Adolesc Health 1997;20:20-26. [PubMed: 9007655]

26. Subar A, Krebs-Smith SM, Cook A, Kahle LL. Dietary sources of nutrients among U.S. children, 1989-1991. Pediatrics 1998;102:913-923. [PubMed: 9755265]

27. Albertson AM, Norton M. Calcium intakes and food sources of the U.S. population and the role of calcium fortification: results from NHANES 1999-2002. FASEB J 2006;20:A620-b.

28. Rafferty KA, Walters G, Heaney RP. Calcium fortificants: overview and strategies for improving calcium nutriture of the U.S. population. J Food Sci 2007;72:R152-R158. [PubMed: 18034744]

29. Yamini S, Juan WY, Marcoe K, Britten P. Impact of using updated food consumption and composition data on selected MyPyramid food group nutrient profiles. J Nutr Educ Behav 2006;38:S136-S152. [PubMed: 17116591]

30. Dwyer J, Picciano MF, Raiten DJ. Members of the Steering Committee. Collection of food and dietary supplement databases for What We Eat in America NHANES. J Nutr 2003;133:624S634S. [PubMed: 12566512] 


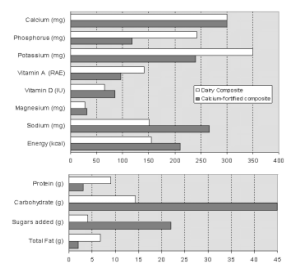

Figure 1.

Nutrient profiles for selected nutrients as consumed by the High Calcium (HC) group, derived from the dairy and calcium-fortified composites and adjusted to equivalent $300 \mathrm{mg}$ calcium levels. Vitamin, mineral, and energy profiles and their respective units of measure are shown in the upper chart; and macronutrient profiles for protein, carbohydrate, added sugar, and fat are shown as gram quantities in the lower chart. 


\section{Table 1}

Examples of changes in calcium fortification levels in selected cereal grain products, 1998-2008

\begin{tabular}{|c|c|c|c|c|}
\hline \multicolumn{5}{|c|}{ Source: USDA Nutrient Database for Standard Reference (SR), Calcium (mg) content of selected foods per common measure ${ }^{a}$} \\
\hline & SR $12(1998)^{a}$ & SR $15(2002)^{a}$ & SR $18(2005)^{a}$ & SR $21(2008)^{a}$ \\
\hline \multicolumn{5}{|l|}{ General Mills ${ }^{\mathrm{TM}}$ brand $b$} \\
\hline Golden Grahams & 14 & 350 & 100 & 100 \\
\hline Total Whole Grain & 258 & 1000 & 1000 & 1000 \\
\hline Basic Four & 310 & 196 & 250 & 250 \\
\hline Lucky Charms & 32 & 100 & 100 & 100 \\
\hline Reese's Puffs & 21 & 100 & 100 & 100 \\
\hline Coco Puffs & 33 & 100 & 100 & 100 \\
\hline Wheaties & 55 & 0 & 20 & 20 \\
\hline \multicolumn{5}{|l|}{ Kelloggs ${ }^{\mathrm{TM}}$ brand $c$} \\
\hline Froot Loops & 100 & 23 & 4 & 23 \\
\hline Apple Jacks & 100 & 8 & 8 & 8 \\
\hline Coco Krispies & 100 & 5 & 5 & 5 \\
\hline Eggo Homestyle Waffle & 150 & 20 & 20 & 20 \\
\hline NutriGrain Cereal Bar $d$ & 20 & 15 & 15 & 200 \\
\hline
\end{tabular}

${ }^{a}$ U.S Department of Agriculture, Agricultural Research Service, 1998, 2002, 2005, 2008. USDA National Nutrient Database for Standard Reference, Release 12,15,18,21. Nutrient Data Laboratory Home Page, http://www.ars.usda.gov/ba/bhnrc/ndl Common measure is the serving size as indicated on the product Nutrition Facts panel

$b_{\text {Fortification of most General Mills }}{ }^{\text {TM }}$ children's cereals with 10\% Daily Value for calcium, effective 1999.

${ }^{c}$ Reformulation of select Kelloggs ${ }^{\mathrm{TM}}$ cereal grain products eliminating calcium fortificant, effective 2001. Calcium values subsequent to 2001 reflect levels of natural calcium and not added calcium.

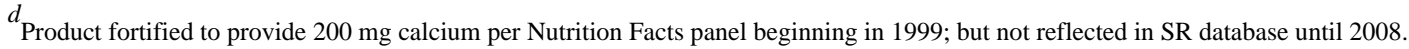


Table 2

Proportionate representation of food components comprising the Dairy and Calcium-Fortified composites for the High Calcium (HC) and Usual Calcium (UC) groups

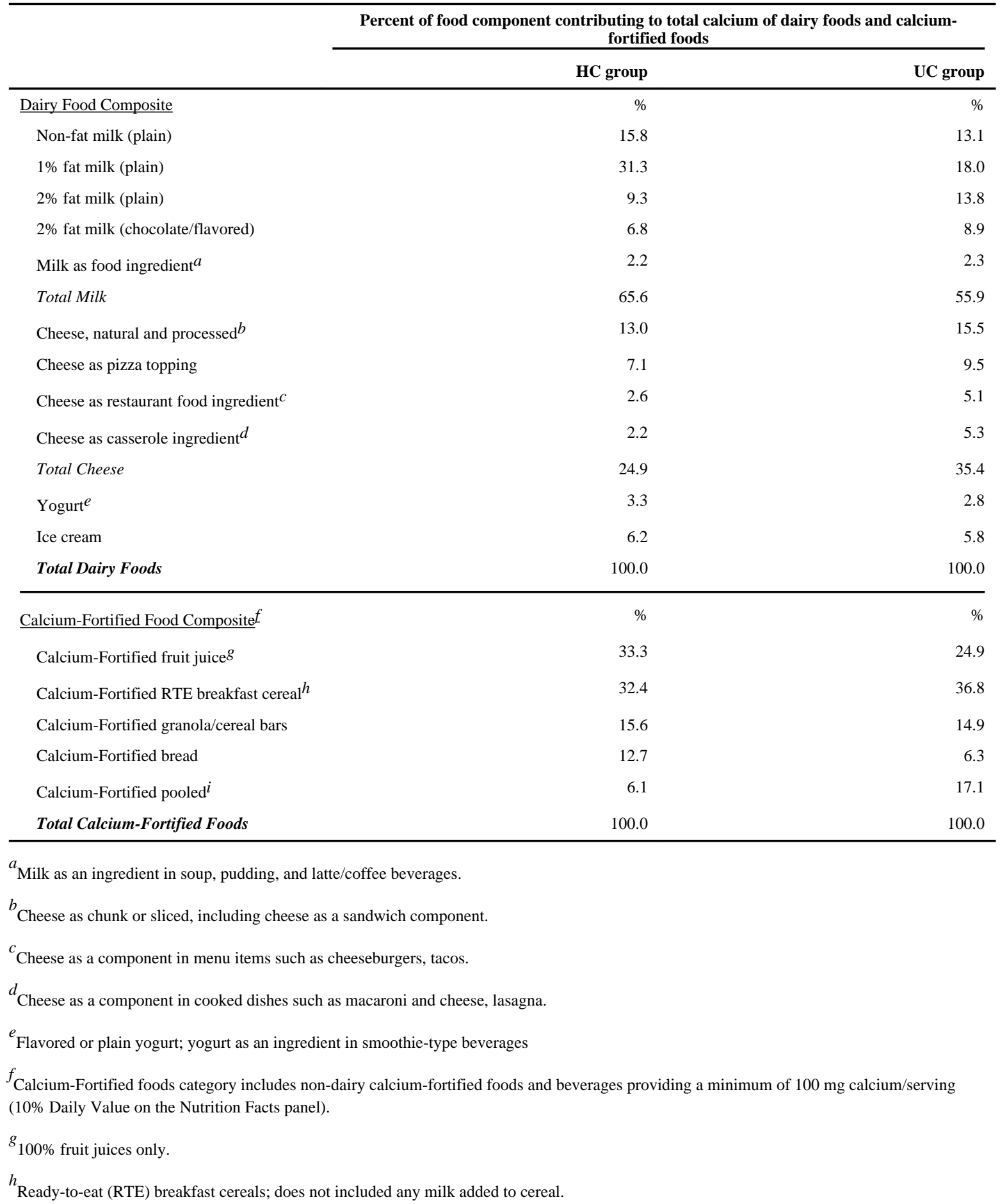

J Nutr Educ Behav. Author manuscript; available in PMC 2012 March 1. 
${ }^{i}$ All other calcium-fortified products consumed in low volume/low frequency during the assessment period. Includes calcium-fortified varieties of waffles, canned/dried pasta products, margarine-type spreads, soy beverages, meal-replacements, and fruit-flavored beverages. 